\title{
miRNA-30a functions as a tumor suppressor by downregulating cyclin E2 expression in castration-resistant prostate cancer
}

\author{
LEI ZHANG ${ }^{1-3^{*}}$, XIAO-WEN ZHANG ${ }^{1-3^{*}}, \mathrm{CHUN} \mathrm{HUI} \mathrm{LIU}^{2,3 *}, \mathrm{KAI} \mathrm{LU}^{1-3}$, \\ YE-QING HUANG ${ }^{2,3}$, YI-DUO WANG ${ }^{1-3}$, LI XING ${ }^{1}$, LI-JIE ZHANG ${ }^{1}$, NING LIU ${ }^{1}$,

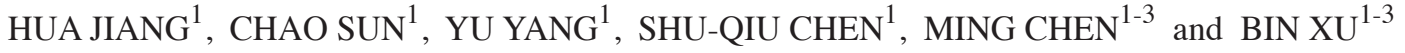 \\ ${ }^{1}$ Department of Urology, Zhongda Hospital; ${ }^{2}$ Surgical Research Center, School of Medicine; \\ ${ }^{3}$ Institute of Urology, Southeast University, Nanjing, Jiangsu 210009, P.R. China
}

Received March 3, 2015; Accepted May 10, 2016

DOI: $10.3892 / \mathrm{mmr} .2016 .5469$

\begin{abstract}
MicroRNAs (miRNAs) act as tumor promoters or tumor suppressors in different human malignancies. In the current study, using an Agilent miRNA microarray, miR-30a was found to be a significantly downregulated miRNA in castration-resistant prostate cancer (CRPC) tissues, compared with androgen-dependent prostate cancer tissues. Aberrant expression of cyclin E2 (CCNE2) has been reported in a variety of types of cancer including prostate cancer, and correlates with clinical outcome. The purpose of the current study was to determine the functions of miR-30a in CRPC cell lines and identify whether CCNE2 was regulated by miR-30a. To analyze the associations between miR-30a and CCNE2 expression levels, pathological specimens were collected, and reverse transcription-quantitative polymerase chain reaction and immunohistochemical staining were conducted. The effect of miR-30a overexpression on CRPC cell lines and the predicted target gene, CCNE2, were evaluated by MTT assay, flow cytometry, tumor formation, luciferase reporter assay and western blotting. miR-30a overexpression resulted in a significant suppression of cell growth in vitro, and reduced tumorigenicity in vivo. miR-30a repressed the expression of CCNE2 through binding to its 3'-untranslated region. CCNE2 was observed to be overexpressed in patients with $\mathrm{CRCP}$ and had an approximately inverse correlation with the level of miR-30a. The results suggest that miR-30a may function as a novel tumor suppressor in CRPC. Its anti-oncogenic activity
\end{abstract}

Correspondence to: Professor Ming Chen or Dr Bin Xu, Department of Urology, Zhongda Hospital, Southeast University, 87 Dingjiaqiao, Gulou, Nanjing, Jiangsu 210009, P.R. China

E-mail: mingchenseu@126.com

E-mail: xb15896450810@126.com

*Contributed equally

Key words: miR-30a, CCNE2, castration-resistant prostate cancer, androgen-dependent prostate cancer, proliferation, metastasis may occur by the reduced expression of a distinct cell cycle protein, CCNE2.

\section{Introduction}

Prostate cancer (PCa) is the most prevalent malignancy in men and the second leading cause of male cancer-associated mortality in the United States (1). Androgens serve vital roles in the initiation and process of PCa. Initially, $\mathrm{PCa}$ responds favorably to hormone deprivation therapy; however, the majority of patients with androgen-dependent PCa (ADPC) inevitably progresses to castration-resistant PCa (CRPC), which has greater malignancy, within 2 years (2). CRPC shows poor response to currently available therapies and ultimately progresses to become terminal. Therefore, studies of the molecular mechanisms involved in PCa progression are of major importance and will aid in the discovery of possible treatment strategies for $\mathrm{PCa}$.

MicroRNAs (miRNAs) are short non-coding, single-stranded RNAs that function by regulating the protein translation and mRNA degradation of their target genes (3). Increasing evidence has indicated that the dysregulation of miRNAs is implicated in human carcinogenesis and cancer progression, indicating that certain miRNAs can function as tumor suppressor genes or oncogenes $(4,5)$. A series of miRNAs have been identified to be aberrantly expressed in $\mathrm{PCa}$ and were suggested to have a functional contribution to $\mathrm{PCa}$ tumorigenesis, including the onco-miRs miR-220/221, miR-125b and miR-21, and the tumor suppressors miR-15a/16, miR-146a and miR-205 (6), which are associated with the regulation of cellular differentiation, proliferation, apoptosis and the acquisition of invasive features and/or androgen independence.

Gene screening was performed using a miRNA chip on ADPC and CRCP tissues, and a miRNA expression database was constructed. In a previous study, miR-30a was identified as being significantly downregulated in CRPC (7), with this result supported by another study which showed miR-30a to be downregulated dramatically in CRPC tissues compared with in ADPC and benign prostatic hyperplasia (BPH) tissues (8). In addition, the cyclin E2 gene (CCNE2) was predicted to be a potential target of miR-30a by computational analysis 
in the present study, and had an approximate crosscurrent of expression in the three groups of tissues. Therefore, based on the previous findings, the aim of the present study was to investigate the function of miR-30a in CRPC cells and confirm whether or not CCNE2 is a direct target of miR-30a.

\section{Materials and methods}

Tissue collection. The BPH samples were collected from transurethral prostatic resection (TURP) specimens from patients treated for BPH. The specimens were histologically confirmed not to contain any prostate cancer cells. For the ADPC tissues, PCa samples were obtained from patients that underwent transrectal prostatic biopsy or radical prostatectomy, and who had not received any previous treatment. Patients were diagnosed with CRPC based on the continual increase in serum prostate-specific antigen levels during maximum androgen deprivation therapy. The CRPC patients underwent TURP due to urinary retention. Each carcinoma specimen was histologically examined for the presence of tumor tissue (>60\%) using hematoxylin and eosin (HE) staining. All the samples were snap frozen in liquid nitrogen and stored at $-80^{\circ} \mathrm{C}$ prior to further analysis. The ethics approval was obtained from the ethics committees at Zhongda Hospital, Southeast University (Nanjing, China) and all samples were collected following the acquisition of informed consent from the patients.

Cell culture. The CRPC cell lines, DU145 and PC3, were obtained from the Type Culture Collection of the Chinese Academy of Sciences (Shanghai, China) and cultured in Dulbecco's modified Eagle's medium (GE Healthcare Life Sciences, Chalfont, UK) supplemented with $10 \%$ fetal bovine serum (FBS; GE Healthcare Life Sciences), and $100 \mu \mathrm{g} / \mathrm{ml}$ streptomycin, $100 \mathrm{U} / \mathrm{ml}$ penicillin, (Gibco; Thermo Fisher Scientific, Inc., Waltham, MA, USA) within a humidified atmosphere containing $5 \% \mathrm{CO}_{2}$ at $37^{\circ} \mathrm{C}$.

Oligonucleotides and cell transfection. All miRNA mimics were designed and synthesised by Shanghai GenePharma Co., Ltd., (Shanghai, China) based on the following sequences: hsa-miR-30a mimics, 5'-UGUAAACAUCCUCGACUG GAAG-3'; and negative control (NC), 5'-UCCAGUCGAGGA UGUUUACAUU-3'. Cell transfection was performed with Lipofectamine 2000 (Invitrogen; Thermo Fisher Scientific, Inc.) according to the manufacturer's protocol. Briefly, $1 \times 10^{6}$ cells were seeded in 6-well plates at $70 \%$ confluence a day prior to transfection. Oligonucleotides formed transfection complexes with Lipofectamine 2000, and were added to cells and incubated for 6-8 $\mathrm{h}$ prior to refreshing the medium.

RNA isolation and reverse transcription-quantitative polymerase chain reaction ( $R T-q P C R)$. Total RNA extraction was performed as previously described (7). Analysis of mature miR-30a expression was performed using TaqMan microRNA assay according to the manufacturer's instructions (Applied Biosystems; Thermo Fisher Scientific, Inc.). Briefly, the reverse transcription reaction was performed in a volume of $15 \mu \mathrm{l}$ containing $5 \mu \mathrm{l}$ total RNA, $3 \mu \mathrm{l} 5 \mathrm{X}$ RT primer and $7 \mu \mathrm{l} \mathrm{RT}$ master mix $(0.15 \mu 1100 \mathrm{mM}$ dNTPs; $1 \mu \mathrm{l}$ MultiScribe reverse transcriptase, $50 \mathrm{U} / \mu \mathrm{l} ; 1.5 \mu \mathrm{l} 10 \mathrm{X}$ reverse transcription buffer;
$0.19 \mu \mathrm{l}$ RNase inhibitor, $20 \mathrm{U} / \mu 1$; and $4.16 \mu \mathrm{l}$ nuclease-free water). For synthesis of cDNA, the reaction mixtures were incubated at $16^{\circ} \mathrm{C}$ for $30 \mathrm{~min}, 42^{\circ} \mathrm{C}$ for $30 \mathrm{~min}$ and $85^{\circ} \mathrm{C}$ for $5 \mathrm{~min}$. The qPCR was performed with a final volume of $20 \mu \mathrm{l}$ containing $1 \mu \mathrm{l}$ 20X TaqMan MicroRNA Assays, $10 \mu \mathrm{l}$ TaqMan Universal PCR Master Mix II (2X), $1.33 \mu$ l RT reaction product and $7.67 \mu \mathrm{l}$ nuclease-free water. The relative miR-30a expression compared with U6 was calculated by the $2^{-\Delta \Delta \mathrm{Cq}}$ method. The reaction for miRNA detection was performed using the following conditions: $95^{\circ} \mathrm{C}$ for $3 \mathrm{~min}, 40$ cycles at $95^{\circ} \mathrm{C}$ for $12 \mathrm{sec}$ and $62^{\circ} \mathrm{C}$ for $40 \mathrm{sec}$. The RT-qPCR reactions were performed using a 7300 Real-Time PCR System (Applied Biosystems; Thermo Fisher Scientific, Inc.). All reactions were run in triplicate.

Cell proliferation assay. Cells transfected with oligonucleotides for over $24 \mathrm{~h}$ were seeded into 96 -well plates (3,000 cells per well). The proliferation of the cells was determined by MTT assay. A total of $20 \mu \mathrm{l}$ MTT $(5 \mathrm{mg} / \mathrm{ml})$ was added to each well at 24, 48 and $72 \mathrm{~h}$, and the cultures were incubated for $4 \mathrm{~h}$ at $37^{\circ} \mathrm{C}$. MTT was carefully aspirated and the purple colored precipitates of formazan were dissolved in $200 \mu 1$ DMSO. The absorbance at $490 \mathrm{~nm}$ was measured using a Model 680 automatic multi-well spectrophotometer (Bio-Rad Laboratories, Inc., Hercules, CA, USA). In total, 5 wells per treatment group were measured for cell proliferation, and all independent treatments were performed in triplicate.

Flow cytometric analysis of the cell cycle and apoptosis. Cells were harvested $48 \mathrm{~h}$ post-transfection. The cell cycle was analyzed by the propidium iodide (PI) staining method and flow cytometry measurements according to the manufacturer's instructions (MultiSciences Biotech Ltd., Hangzhou, China). Apoptosis was analyzed by the annexin V-fluorescein isothiocyanate (FITC) plus PI staining method according to the manufacturer's instructions (Ubio Biotechnology Systems Pvt, Ltd., Jinan, China). The treated cells were analyzed by flow cytometry on a FACSCalibur system (BD Biosciences, Franklin Lakes, NJ, USA). A minimum of 20,000 cells were acquired for each sample. The experiments were performed in triplicate.

Cell migration and invasion assays. For the migration assays, $1 \times 10^{5}$ cells in serum-free medium were placed in the upper chamber of the Transwell with $8 \mu \mathrm{m}$ pore size polycarbonate membrane filters (BD Biosciences). For the invasion assays, matrigel (BD Biosciences) was applied to the polycarbonate membrane filters of the upper chamber, following which $1 \times 10^{5}$ cells in serum-free medium were seeded according to the manufacturer's protocol. To the lower chamber, the same medium was added, containing $10 \%$ FBS and the chamber was incubated for $24 \mathrm{~h}$ at $37^{\circ} \mathrm{C}$. Following this, the cells remaining on the upper membrane were removed using a cotton-tip applicator, and the cells on the lower surface of the membrane were fixed with methanol and stained with crystal violet. Cells were quantified by counting five random high-powered fields. All of the experiments were performed in triplicate.

Tumor formation assay in a nude mouse model. Immunodeficient BALB/C nu/nu male mice ( $\mathrm{n}=3 ; 5$ weeks 
old) were obtained from the Shanghai Laboratory Animal Centre (Shanghai, China). The animal experiments were undertaken in accordance with the National Institute of Health Guide for the Care and Use of Laboratory Animals (9) and were approved by the ethics committee of Zhongda Hospital, Southeast University (Nanjing, China). PC3 cells transfected with oligonucleotides for $48 \mathrm{~h}$ were harvested and the tumor formation assay conducted as previously described (10). Tumors were formalin fixed immediately following harvesting and paraffin embedded.

Immunohistochemical staining (IHC). The IHC kit (NeoBioscience Technology Co., Ltd., Beijing, China) was used for IHC staining. The paraffin embedded clinical specimens and xenograft tumors were processed according to the manufacturer's instructions. Briefly, 4- $\mu \mathrm{m}$ thick sections of the sample tissues were deparaffinized and rehydrated, then heat-based antigen retrieval $\left(20 \mathrm{~min}\right.$ at $\left.95^{\circ} \mathrm{C}\right)$ was conducted, followed by endogenous peroxidase blocking with $3 \%$ hydrogen peroxide, and nonspecific protein blocking with reagent $\mathrm{A}$ from the kit (10\% goat serum). Sections were incubated at $37^{\circ} \mathrm{C}$ for $1 \mathrm{~h}$ with the following primary antibodies purchased from Abcam (Cambridge, MA, USA): Rabbit monoclonal anti-human CCNE2 (1:250; \#ab40890) and rabbit polyclonal anti-human Ki67 (1:100; \#ab66155) antibodies. This was followed by incubation with reagent B (biotinylated goat anti rabbit $\mathrm{IgG}$ ) for $10 \mathrm{~min}$ and reagent $\mathrm{C}$ (streptavidin-labeled horseradish peroxidase) for $10 \mathrm{~min}$ at room temperature. The reaction was visualized by DAB developer mixed from reagent D, E and F. An Eclipse E600 microscope (Nikon Corporation, Tokyo, Japan) was used for visualization. For the negative controls, sections were treated following the same procedure except that they were incubated with Tris-buffered saline (TBS) without primary antibody.

Plasmid construction and luciferase assays. The 3'-UTR segment of CCNE2 mRNA containing the miR-30a binding site was amplified by PCR from human DNA extracted from peripheral blood of the human participants. The primers used contained the following restriction sites: Forward, 5'-CCG CTCGAGCACAAGTTACACTGCCATTC-3' (XhoI) and reverse, 5'-CTTGCGGCCGCGCTATAGCAGCTATAG ATA-3' (NotI). The PCR product was cloned into the XhoI and NotI restriction sites downstream of the open reading frame of luciferase in a psiCHECK-2 Vector (Promega Corporation, Madison, WI, USA) to generate the CCNE2 3 '-UTR reporter. For the reporter assays, $1 \times 10^{6}$ cells were seeded in 24-well plates and co-transfected at $70 \%$ confluence with $0.25 \mu \mathrm{g}$ of CCNE2 3'UTR or control reporter plasmid and 25 pmol miR-30a or NC mimics using Lipofectamine 2000. Each transfection was performed in triplicate and luciferase activity was assessed $48 \mathrm{~h}$ after transfection using dual luciferase assays (Promega Corporation). Firefly luciferase activity was normalized to Renilla luciferase activity accordingly.

Western blotting. Cells were transfected with oligonucleotides, then harvested on ice $72 \mathrm{~h}$ later. Protein isolation and western blotting analyses were conducted as previously described (10). Cells were lysed with radioimmunoprecipitation buffer
(Beyotime Institute of Biotechnology, Haimen, China) then total protein concentrations were determined by bicinchoninic acid assay (Beyotime Institute of Biotechnology). Protein samples $(30 \mu \mathrm{g})$ were separated by $10 \%$ SDS-PAGE (Beyotime Institute of Biotechnology) at $80 \mathrm{~V}$ for $30 \mathrm{~min}$ then $100 \mathrm{~V}$ for $1.5 \mathrm{~h}$. Electrophoresed proteins were transferred to a polyvinylidene difluoride membrane (EMD Millipore, Billerica, MA, USA) and subsequently blocked with $5 \%$ skimmed milk (BioSharp, Hefei, China) at room temperature for $1 \mathrm{~h}$. The membranes were incubated with the abovementioned CCNE2 $(1: 1,000)$ antibody, or rabbit polyclonal anti-human GAPDH antibody (1:1,000; \#sc25778; Santa Cruz Biotechnology, Inc., Dallas, TX, USA) in $5 \%$ skimmed milk overnight at $4^{\circ} \mathrm{C}$. The blots were washed 3 times with TBS (pH 7.6, 20 mM Tris- $\mathrm{HCl}$, $137 \mathrm{mM} \mathrm{NaCl}$ ) with $0.01 \%$ Tween 20 , incubated with horseradish peroxidase-labeled goat anti-rabbit secondary antibody (1:3,000; \#ZB2301; Zhongshan Golden Bridge Biotechnology Co., Ltd., Beijing, China) at $37^{\circ} \mathrm{C}$ for $1 \mathrm{~h}$, and visualized using Immobilon Western Chemilum HRP Substrate (EMD Millipore). Blots were exposed to the film $(5 \times 7$ inch; Carestream Health Co., Ltd., Xiamen, China) for $5 \mathrm{~min}$ in X-ray film cassette (Yuehua Medical Instrument Factory Co., Ltd., Shantou, China), and then developed and fixed (Beyotime Institute of Biotechnology). Protein levels were determined by normalization against GAPDH.

Bioinformatic and statistical analysis. The miRNA target predicting algorithms miRDB (www.mirdb.org/miRDB/) and TargetScan (www.targetscan.org/) were used to predict miRNAs targeting CCNE2 and the binding regions. Data in the present study was obtained from at least three independent experiments and presented as the mean \pm standard error. The correlation between the expression of miR-30a and CCNE2 was examined by Spearman correlation analysis. Group means were compared by Student's $t$-test. Expression of miR-30a and CCNE2 in three groups were analyzed by analysis of variance followed by Tukey's multiple comparisons test. All statistical analyses were performed using SPSS software, version 16.0 (SPSS, Inc., Chicago, IL, USA). $\mathrm{P}<0.05$ was considered to indicate a statistically significant difference.

\section{Results}

Expression of miR-30a and CCNE2 and the correlation between miR-30a and CCNE2 in the three patient groups. Microarray data from a previous study indicated that miR-30a expression in the CRPC tissues was lower compared with the ADPC tissues (7). To verify these microarray results, the current study assessed the miR-30a expression levels in 5 BPH, 5 ADPC and 5 CRPC tissues by RT-qPCR. Compared with the ADPC and BPH tissues, miR-30a expression was markedly downregulated in CRPC tissues $(\mathrm{P}<0.05)$, while no significant difference was observed between the ADPC and BPH tissues (Fig. 1A). Immunohistochemistry was conducted to detect CCNE2 protein expression in the BPH, ADPC and CRPC tissues. The results showed that CCNE2 was expressed strongly in 3 of 5 CRPC tissues $(\mathrm{P}<0.05)$, while no significant expression was observed in ADPC or BPH tissues (Fig. 1B). In agreement with miR-30a inhibition of CCNE2 in cultured 
A
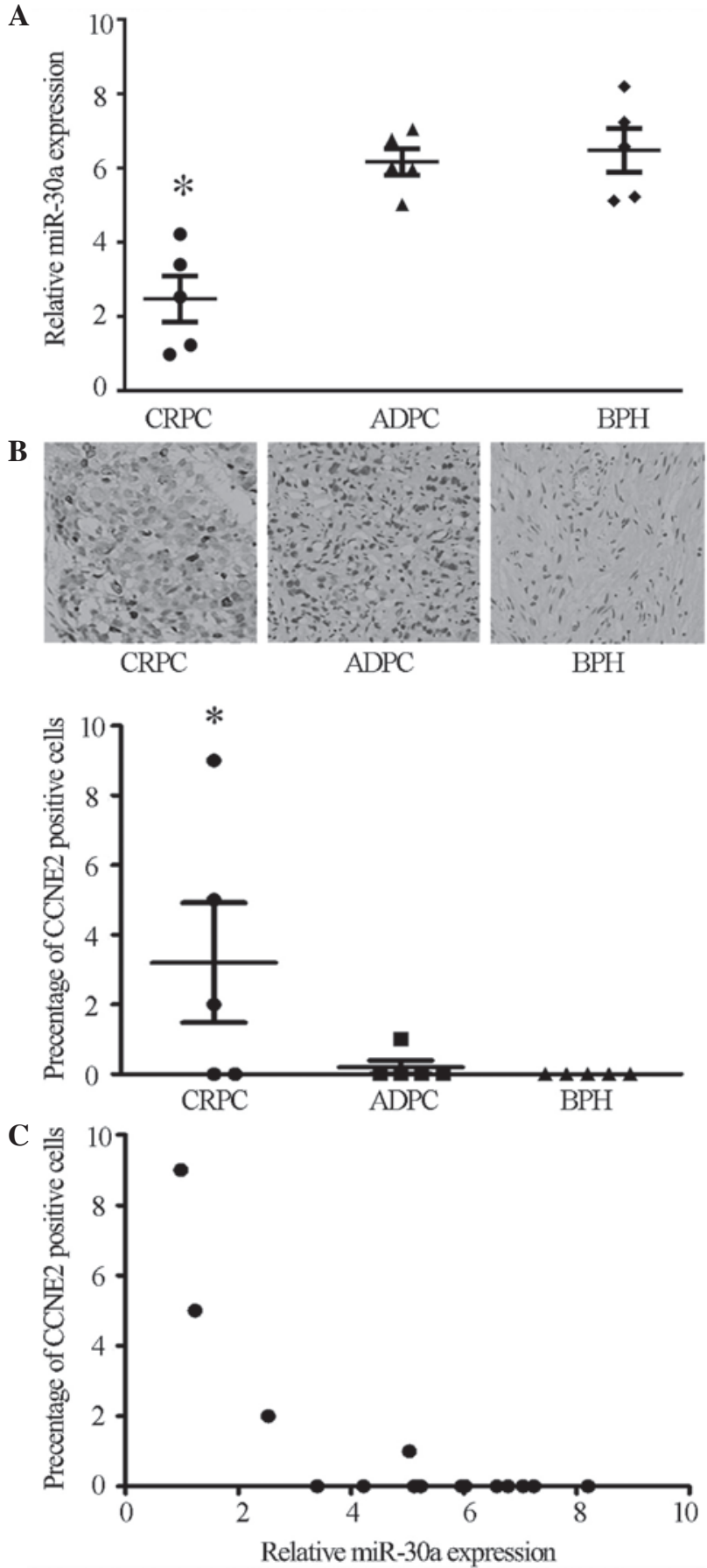

Figure 1. Expression of miR-30a and CCNE2 and the correlation between miR-30a and CCNE2 in three groups of patients. (A) miR-30a expression in human CRPC, ADPC and BPH tissues was measured using reverse transcription-quantitative polymerase chain reaction. (B) Representative immunohistochemical staining of CCNE2 in tissues from the three groups. Magnification, x200. Quantification of CCNE2 expression in human CRPC, ADPC and BPH tissues. (C) The association between miR-30a and CCNE2 expression was investigated using Spearman rank correlation analysis $(\mathrm{P}<0.05, \mathrm{r}=-0.72) .{ }^{*} \mathrm{P}<0.05 . \mathrm{n}=5$. miR, microRNA; CCNE2, cyclin E2; CRPC, castration-resistant prostate cancer; ADPC, androgen-dependent prostate cancer; $\mathrm{BPH}$, benign prostate hyperplasia.

cells, the expression levels of miR-30a negatively correlated with CCNE2 expression in the prostate samples $(\mathrm{P}<0.05$, $\mathrm{r}=-0.72$; Fig. 1C). Taken together, these data suggest a potential interaction between miR-30a and CCNE2 in the progression of ADPC to CRPC.
CCNE2 is a direct target of miR-30a. A bioinformatic search (TargetScan, miRDB) was performed for putative targets of miR-30a, and miR-30a was identified as being able to bind to target sequences located in nucleotides 212-218 and 475-481 of the 3'-UTR of CCNE2 mRNA (Fig. 2A). To ascertain the direct miRNA-target interaction, the CCNE2 3'-UTR was cloned into a luciferase reporter cloning site in a psiCHECK-2 dual luciferase vector. With increasing miR-30a levels, the luciferase activities were markedly reduced $(\mathrm{P}<0.05$; Fig. 2B). Furthermore, western blotting indicated that the levels of CCNE2 were reduced by treatment with miR-30a mimics $(\mathrm{P}<0.05$; Fig. 2C).

miR-30a suppresses tumorigenicity and metastasis in vitro. To investigate the function of miR-30a in CRPC, miR-30a expression was restored in CRPC cell lines. DU145 and PC3 cells were transfected with miR-30a or NC, following which functional assays were conducted. The MTT assay, as shown in Fig. 3A, demonstrated that the restoration of miR-30a expression significantly inhibited the growth of CRPC cells at 48 and $72 \mathrm{~h}(\mathrm{P}<0.05)$. To assess the role of miR-30a in cell cycle progression, miR-30a expression was restored in DU145 and PC 3 cells. Compared with NC transfectants, flow cytometric analysis of PI-stained cells transfected with miR-30a demonstrated a $\mathrm{G}_{1}$ accumulation $48 \mathrm{~h}$ following transfection $(\mathrm{P}<0.05 ;$ Fig. 3B). Annexin V-FITC/PI stained cells transfected with miR-30a showed a higher rate of apoptosis $(\mathrm{P}<0.05$; Fig. 3C). These results indicate that miR-30a induces cell cycle arrest and apoptosis in CRPC cells. Transwell migration and invasion assays showed that the migration and invasion of miR-30a-transfected cells was reduced compared with the $\mathrm{NC}$ transfectants $(\mathrm{P}<0.05$; Fig. $4 \mathrm{~A}$ and $\mathrm{B})$. This suggests that the restoration of miR-30a expression suppresses the tumorigenicity and metastasis of CRPC cells in vitro.

miR-30a suppresses tumor growth in vivo. To investigate the effect of miR-30a on tumorigenicity and tumor progression in vivo, miR-30a and NC-transfected $\mathrm{PC} 3$ cells were subcutaneously injected into either flank of nude mice. As predicted, miR-30a reduced the volume of tumors formed from PC3 cells $(\mathrm{P}<0.05$; Fig. 5A). Furthermore, miR-30a reduced Ki-67 and CCNE2 staining in tumor xenografts $(\mathrm{P}<0.05$; Fig. $5 \mathrm{~B}$ and $\mathrm{C})$, suggesting that the miR-30a/CCNE2 axis may reduce tumorigenicity and tumor progression in a nude mouse model.

\section{Discussion}

Understanding the underlying molecular mechanisms of prostate tumorigenesis and cancer progression is crucial to improve clinical treatment and the management of patients with CRPC. In previous studies, it was reported that the loss of miR-146a is a critical mechanism for the overexpression of epidermal growth factor (EGF) receptor in CRPC (10), and miR-361-5p can act as a tumor suppressor by targeting signal transducer and activator of transcription 6 in CRPC (7). Based on the preliminary microarray analysis, miR-30a was focused upon for subsequent investigation, which indicated it was downregulated in CRPC tissues. miR-30a expression was markedly downregulated in CRPC tissues compared with the ADPC and $\mathrm{BPH}$ tissues, however, there was no significant difference 
A

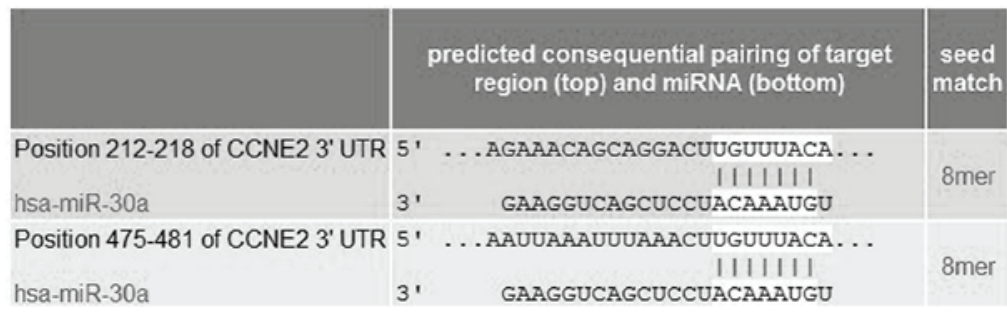

B

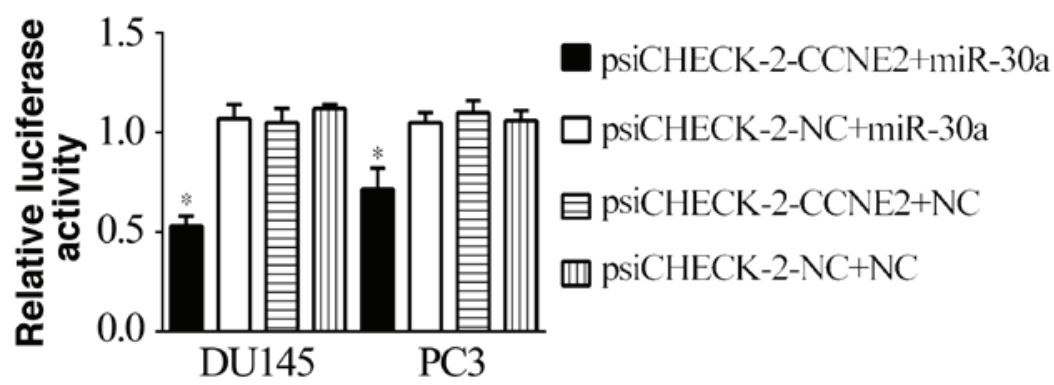

C
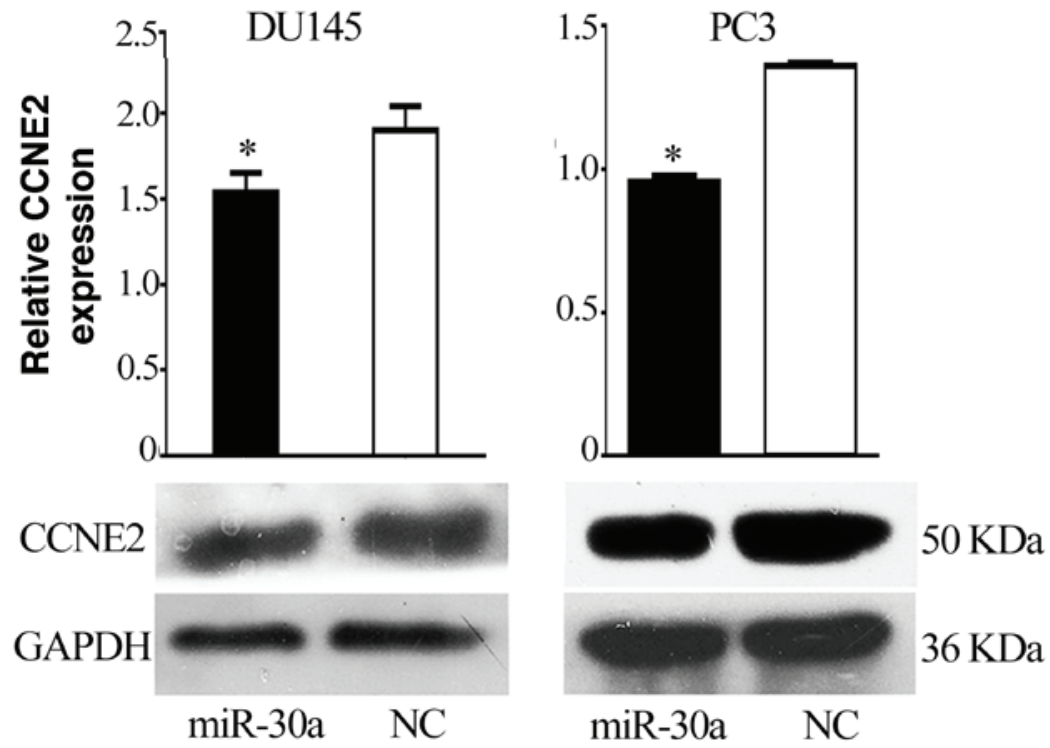

Figure 2. CCNE2 is a direct target of miR-30a in prostate cancer cells. (A) The TargetScan database showed that miR-30a may bind to two target sequences located at nucleotides 212-218 and 475-481 of the 3'-UTR of the CCNE2 mRNA. (B) The CCNE2 3'-UTR was cloned into the luciferase reporter cloning site in a psiCHECK-2 dual luciferase vector. The increased expression levels of miR-30a resulted in a reduction in luciferase activity. (C) The protein expression levels of CCNE2 were reduced following treatment with miR-30a mimics, as measured by western blotting analysis. * $<<0.05$. CCNE2, cyclin E2; miR, microRNA; UTR, untranslated region; NC, negative control; GAPDH, glyceraldehyde 3-phosphate dehydrogenase.

between the ADPC and BPH tissues, which is consistent with previous reports $(8,11)$. These results suggest that miR-30a may serve a role in the progression of ADPC to CRPC. Previous studies have reported miR-30a to be a tumor suppressor in renal cell carcinoma (12), non-small cell lung cancer (13), breast cancer (14) and colorectal carcinoma (15), however, a tumor inducer in glioma (16). These contradictory reports indicate that there may be disease-specific modulation of miR-30a. For prostate cancer, there has been a single previous study, which indicated that miR-30 suppresses epithelial-to-mesenchymal transition (EMT) phenotypes and inhibits migration and invasion in prostate cancer cells by connecting EGF/Src signal to Ets-related gene and EMT (17). In the present study, functional analyses showed that the restoration of miR-30a expression in CRPC cells significantly suppressed proliferation, migration and invasion ability, induced cell cycle arrest and apoptosis in vitro, and in addition, reduced tumorigenicity and tumor progression in vivo. Furthermore, the present study reported another oncogene, CCNE2, as a target gene of miR-30a in CRPC.

Cyclin E is composed of cyclin E1 and E2, which are encoded by separate genes located at chromosomes $19 \mathrm{q} 12$ (CCNE1) and 8q22.1 (CCNE2) in humans, however, they share high sequence identity and functional redundancy (18). Cyclin $E$ activates $\mathrm{Cdk} 2$ in late $\mathrm{G}_{1}$ phase, driving the transition from $\mathrm{G}_{1}$ to $\mathrm{S}$ phase, with $\mathrm{Cdk} 2$ phosphorylating the $\mathrm{Rb}$ protein and other targets necessary for the initiation of DNA replication (19). Unlike CCNE1, which is expressed in most proliferating normal and tumor cells, CCNE2 levels are low to undetectable in nontransformed cells, with levels increasing 
A

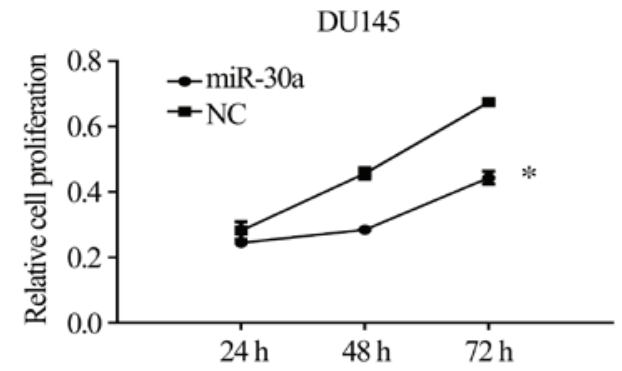

B
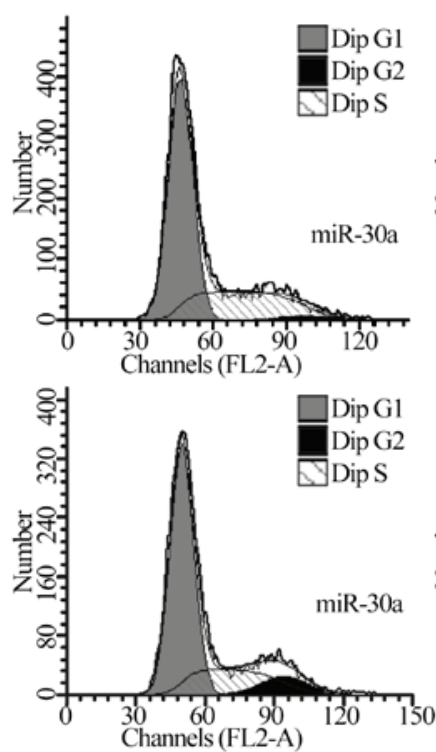

C
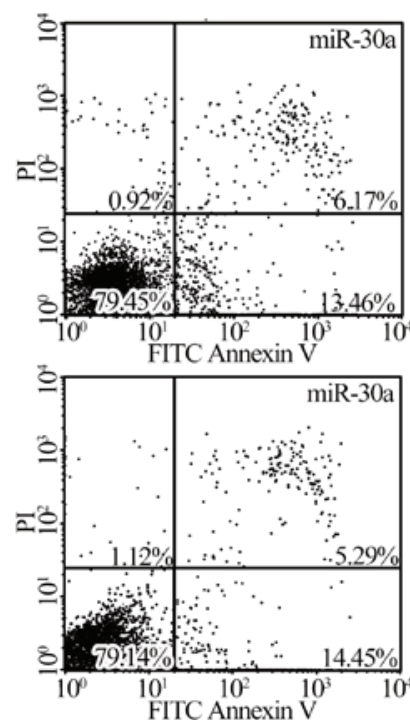
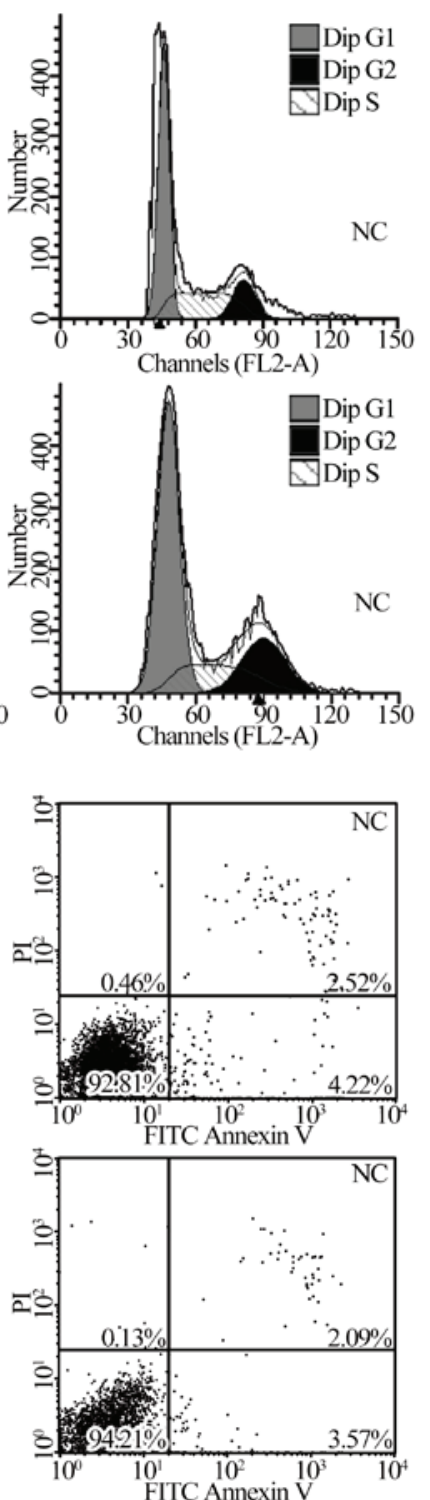

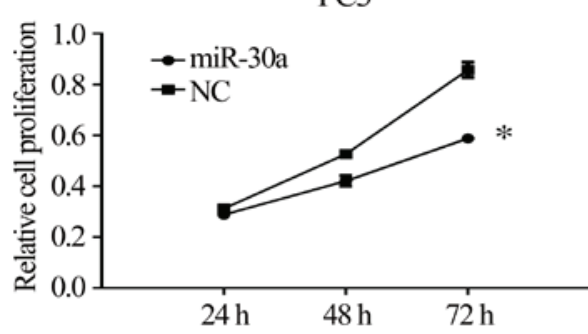

DU145
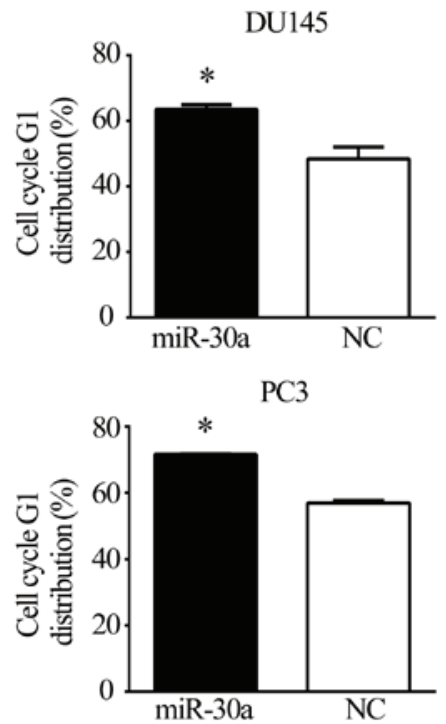

DU145
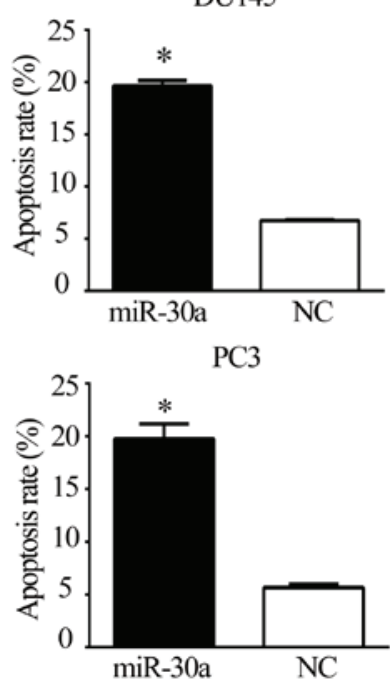

Figure 3. miR-30a suppresses cell proliferation and induces cell cycle arrest and apoptosis. (A) An MTT assay was performed on cells transfected with miR-30a or NC mimics, indicating that the proliferation of cells transfected with miR-30a was reduced. (B) miR-30a induces $\mathrm{G}_{1}$ cell cycle arrest in DU145 and PC3 cells, as evaluated by flow cytometry. (C) Annexin V-FITC/PI-stained cells transfected with miR-30a showed a higher rate of apoptosis, as evaluated by flow cytometry. Data are presented as the mean \pm standard error, from a minimum of three independent experiments. ${ }^{*}<<0.05$. miR, microRNA; $\mathrm{NC}$, negative control; FITC, fluorescein isothiocyanate; PI, propidium iodide; Dip, diploid.

significantly in tumor-derived cells (20), suggesting that mechanisms distinct from CCNE1 induce tumorigenesis and progression (21). Previous studies indicate that CCNE2 overexpression is associated with pathogenesis (22), endocrine resistance (23), metastasis and reduced survival (24) in breast cancer. A previous study examined $\mathrm{PCa}$ array data (25) and showed higher expression levels of CCNE2 probe sets in metastatic samples compared with benign and localized samples. This is supported by results of an independent analysis using Oncomine (26). Furthermore, CCNE2 is phosphatase and tensin homolog-regulated and is associated with cell cycle arrest in $\mathrm{G}_{1}$ phase and metastasis in PCa (27). In the current study, it was observed that CCNE2 was overexpressed in patients with $\mathrm{CRCP}$, and had an inverse correlation with the 
A
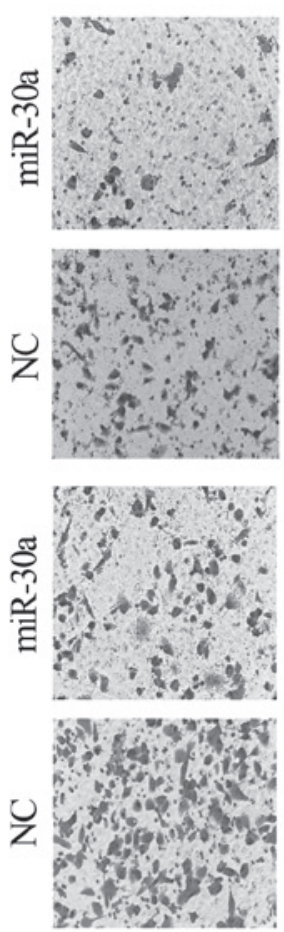

B
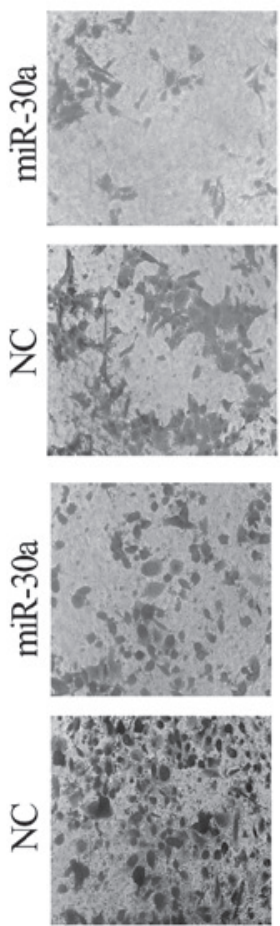

DU145

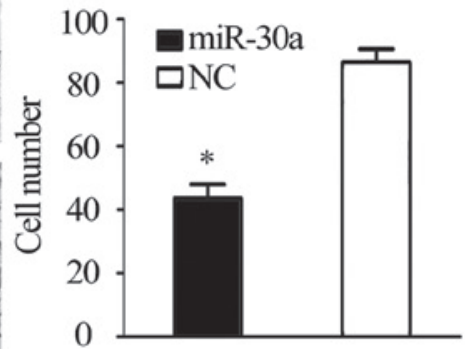

PC3

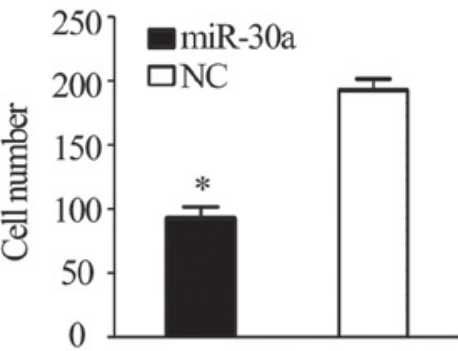

DU145

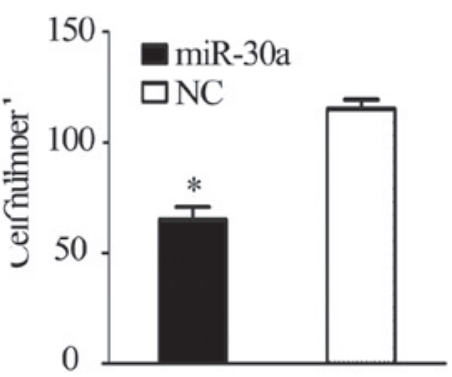

PC3

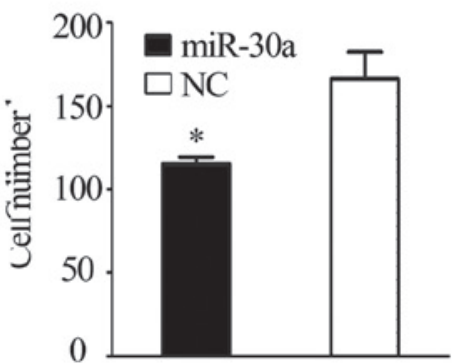

A

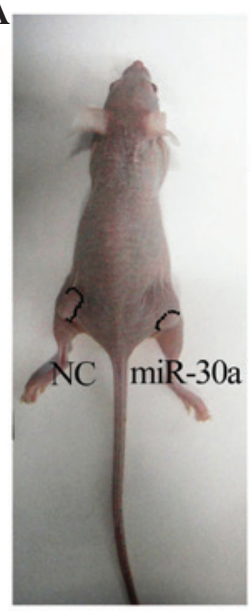

B
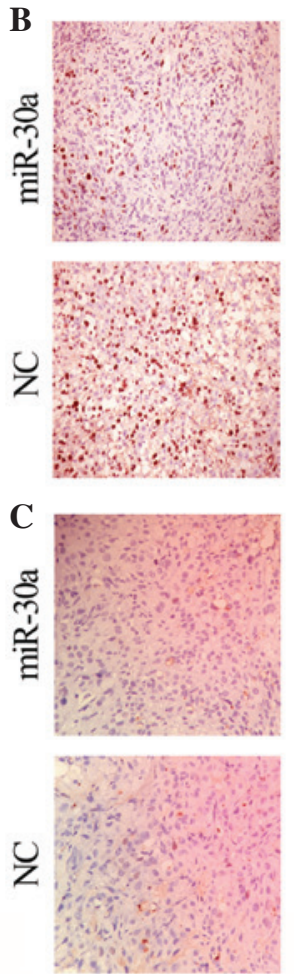

NC

miR-30a
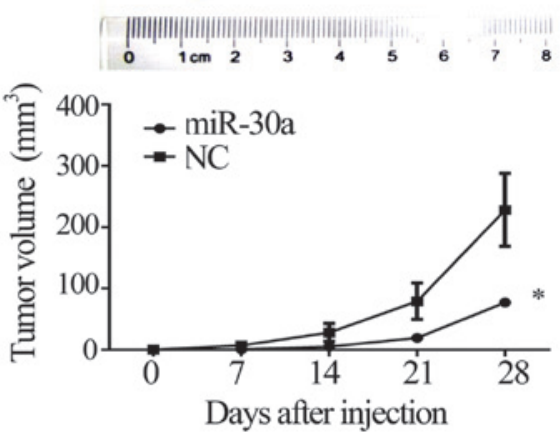

Ki-67

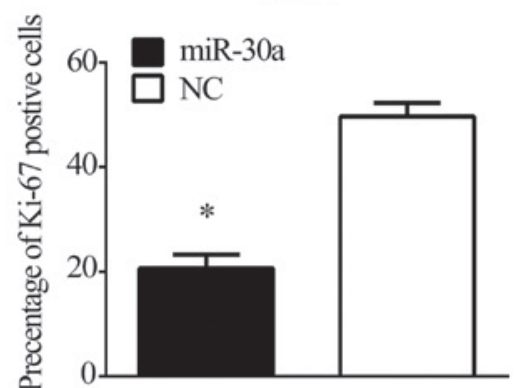

CCNE2

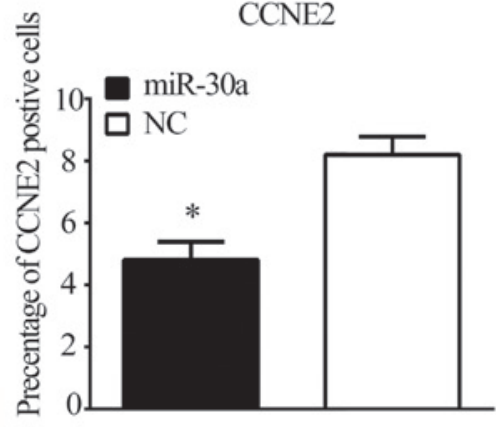

Figure 4. miR-30a inhibited cell (A) migration and (B) invasion in the Transwell assay. Data are presented as the mean \pm standard error, from a minimum of three independent experiments. ${ }^{*} \mathrm{P}<0.05$. Magnification, $\mathrm{x} 200$. miR, microRNA; NC, negative control.

level of miR-30a. Furthermore, CCNE2 was identified as a direct target of miR-30a. Overexpression of miR-30a reduced the malignant progression of PCa cells and reduced the expression of CCNE2 in vitro and in vivo.

In conclusion, these data provide evidence that miR-30a, which is frequently downregulated in CRPC, supports the multi-step process of CRPC development via modulating CCNE2 expression, which in turn alters cancer development

Figure 5. miR-30a reduced the growth of PC3 cell xenografts in vivo. (A) The tumor volume of the miR-30a group was significantly reduced compared with the NC group. (B) miR-30a reduced Ki-67 immunostaining in tumors. (C) miR-30a reduced CCNE2 immunostaining in tumors. ${ }^{*} \mathrm{P}<0.05$. Magnification, (B and C) x200. miR, microRNA; NC, negative control; CCNE2, cyclin E2.

and progression. Therefore, miR-30a and CCNE2 may be regarded as potential targets for CRPC therapy.

\section{Acknowledgements}

The present study was supported by the National Natural Science Foundation of China (grant nos. 81370849, 81300472, 81070592 and 81202034), the Natural Science Foundation of Jiangsu Province (grant nos. BK2013032 and BK2012336), Nanjing City (grant no. 201201053) and Southeast University (grant no. 3290002402), and the Science Foundation of the Ministry of Education of China (grant no. 20120092120071). 


\section{References}

1. Siegel R, Ma J, Zhou Z and Jemal A: Cancer statistics, 2014. CA Cancer J Clin 64: 9-29, 2014.

2. Feldman BJ and Feldman D: The development of androgen independent prostate cancer. Nat Rev Cancer 1: 34-45, 2001.

3. Lai EC: MicroRNAs are complementary to $3^{\prime}$ UTR sequence motifs that mediate negative post-transcriptional regulation. Nat Genet 30: 363-364, 2002.

4. Garzon R, Calin GA and Croce CM: MicroRNAs in cancer. Annu Rev Med 60: 167-179, 2009.

5. Zhang L, Xul B, Chen S, Lu K, Liu C, Wang Y, Zhao Y, Zhang X, Liu D and Chen M: The complex roles of microRNAS in the metastasis of renal cell carcinoma. J Nanosci Nanotechnol 13 3195-3203, 2013

6. Coppola V, De Maria R and Bonci D: MicroRNAs and prostate cancer. Endocr Relat Cancer 17: F1-F17, 2010.

7. Liu D, Tao T, Xu B, Chen S, Liu C, Zhang L, Lu K, Huang Y, Jiang L, Zhang X, et al: MiR-361-5p acts as a tumor suppressor in prostate cancer by targeting signal transducer and activator of transcription-6(STAT6). Biochem Biophys Res Commun 445: 151-156, 2014.

8. Porkka KP, Pfeiffer MJ, Waltering KK, Vessella RL, Tammela TL and Visakorpi T: MicroRNA expression profiling in prostate cancer. Cancer Res 67: 6130-6135, 2007.

9. National Research Council: Guide for the care and use of laboratory animals. 7th edition. National Academy Press, Washington DC, 1996.

10. Xu B, Wang N, Wang X, Tong N, Shao N, Tao J, Li P, Niu X, Feng N, Zhang L, et al: MiR-146a suppresses tumor growth and progression by targeting EGFR pathway and in a p-ERK-dependent manner in castration-resistant prostate cancer. Prostate 72: 1171-1178, 2012.

11. Ozen M, Creighton CJ, Ozdemir M and Ittmann M: Widespread deregulation of microRNA expression in human prostate cancer. Oncogene 27: 1788-1793, 2008.

12. Huang QB, Ma X, Zhang X, Liu SW, Ai Q, Shi TP, Zhang Y, Gao Y, Fan Y and Ni D, et al: Down-Regulated miR-30a in clear cell renal cell carcinoma correlated with tumor hematogenous metastasis by targeting angiogenesis-specific DLL4. PLoS One 8: e67294, 2013.

13. Kumarswamy R,Mudduluru G,Ceppi P,MuppalaS,KozlowskiM, Niklinski J, Papotti M and Allgayer H: MicroRNA-30a inhibits epithelial-to-mesenchymal transition by targeting Snail and is downregulated in non-small cell lung cancer. Int J Cancer 130: 2044-2053, 2012.

14. Fu J, Xu X, Kang L, Zhou L, Wang S, Lu J, Cheng L, Fan Z, Yuan B, Tian P, et al: MiR-30a suppresses breast cancer cell proliferation and migration by targeting Eya2. Biochem Biophys Res Commun 445: 314-319, 2014.
15. Baraniskin A, Birkenkamp-Demtroder K, Maghnouj A, Zöllner H, Munding J, Klein-Scory S, Reinacher-Schick A, Schwarte-Waldhoff I, Schmiegel W and Hahn SA: MiR-30a-5p suppresses tumor growth in colon carcinoma by targeting DTL. Carcinogenesis 33: 732-739, 2012.

16. Jia Z, Wang K, Wang G, Zhang A and Pu P: MiR-30a-5p antisense oligonucleotide suppresses glioma cell growth by targeting SEPT7. PLoS One 8: e55008, 2013.

17. Kao CJ, Martiniez A, Shi XB, Yang J, Evans CP, Dobi A, deVere White RW and Kung HJ: MiR-30 as a tumor suppressor connects EGF/Src signal to ERG and EMT. Oncogene 33: 2495-2503, 2014.

18. Caldon CE and Musgrove EA: Distinct and redundant functions of cyclin E1 and cyclin E2 in development and cancer. Cell Div 5: 2, 2010.

19. Hwang HC and Clurman BE: Cyclin E in normal and neoplastic cell cycles. Oncogene 24: 2776-2786, 2005.

20. Gudas JM, Payton M, Thukral S, Chen E, Bass M, Robinson MO and Coats S: Cyclin E2, a novel G1 cyclin that binds Cdk2 and is aberrantly expressed in human cancers. Mol Cell Biol 19: 612-622, 1999.

21. Caldon CE, Sergio CM, Burgess A, Deans AJ, Sutherland RL and Musgrove EA: Cyclin E2 induces genomic instability by mechanisms distinct from cyclin E1. Cell Cycle 12: 606-617, 2013.

22. Payton M, Scully S, Chung G and Coats S: Deregulation of cyclin E2 expression and associated kinase activity in primary breast tumors. Oncogene 21: 8529-8534, 2002.

23. Caldon CE, Sergio CM, Kang J, Muthukaruppan A, Boersma MN, Stone A, Barraclough J, Lee CS, Black MA, Miller LD, et al: Cyclin E2 overexpression is associated with endocrine resistance but not insensitivity to CDK2 inhibition in human breast cancer cells. Mol Cancer Ther 11: 1488-1499, 2012.

24. Sieuwerts AM,Look MP, Meijer-van Gelder ME,Timmermans M, Trapman AM, Garcia RR, Arnold M, Goedheer AJ, de Weerd V, Portengen $\mathrm{H}$, et al: Which cyclin E prevails as prognostic marker for breast cancer? Results from a retrospective study involving 635 lymph node-negative breast cancer patients. Clin Cancer Res 12: 3319-3328, 2006.

25. Varambally S, Yu J,Laxman B, Rhodes DR, Mehra R, Tomlins SA, Shah RB, Chandran U, Monzon FA, Becich MJ, et al: Integrative genomic and proteomic analysis of prostate cancer reveals signatures of metastatic progression. Cancer Cell 8: 393-406, 2005.

26. Yu YP, Landsittel D, Jing L, Nelson J, Ren B, Liu L, McDonald C, Thomas R, Dhir R, Finkelstein S, et al: Gene expression alterations in prostate cancer predicting tumor aggression and preceding development of malignancy. J Clin Oncol 22: 2790-2799, 2004.

27. Wu Z, Cho H, Hampton GM and Theodorescu D: Cdc6 and cyclin E2 are PTEN-regulated genes associated with human prostate cancer metastasis. Neoplasia 11: 66-76, 2009. 